Computer simulations of confined colloidal systems at the air/water interface

This content has been downloaded from IOPscience. Please scroll down to see the full text. 2002 J. Phys.: Condens. Matter 144795

(http://iopscience.iop.org/0953-8984/14/19/306)

View the table of contents for this issue, or go to the journal homepage for more

Download details:

IP Address: 148.234.36.245

This content was downloaded on 28/01/2016 at 17:39

Please note that terms and conditions apply. 


\title{
Computer simulations of confined colloidal systems at the air/water interface
}

\author{
S J Mejía-Rosales ${ }^{1}$, A Gil-Villegas ${ }^{2,4}$, B I Ivlev ${ }^{1,3}$ and J Ruiz-Garcia ${ }^{1,4}$ \\ ${ }^{1}$ Instituto de Física 'Manuel Sandoval Vallarta', Universidad Autónoma de San Luis Potosí, \\ Alvaro Obregon 64, 78000 San Luis Potosí, SLP, Mexico \\ ${ }^{2}$ Instituto de Física, Universidad de Guanajuato, 37150 León, Guanajuato, Mexico \\ ${ }^{3}$ Department of Physics and Astronomy, University of South Carolina, Columbia, \\ SC 29208, USA \\ E-mail: jaime@dec1.ifisica.uaslp.mx and gil@ifug1.ugto.mx
}

Received 22 November 2001, in final form 15 February 2002

Published 2 May 2002

Online at stacks.iop.org/JPhysCM/14/4795

\begin{abstract}
We present Monte Carlo simulations in the $N V T$ ensemble for spherical particles of diameter $\sigma$ interacting via a hard-core potential with a squareshoulder (SS) repulsive barrier, a square-well (SW) attraction, and a second SS repulsion. This discrete potential is used to mimic the pair interaction in confined colloidal systems at the air/water interface. The SW attraction represents a secondary minimum for large interparticle distances, and the SS repulsion is a shallow secondary maximum after the secondary minimum. The effect of the SS range $\lambda_{r} \sigma$ is studied for the cases $\lambda_{r}=0,6$, and 7. The simulation results for the last two cases indicate the important role of the presence of the secondary maximum in the interaction potential, since they are able to reproduce the main features observed in colloidal particles trapped at the air/water interface, such as clustering, chain formation, foams, and the presence of voids.
\end{abstract}

\section{Introduction}

Recent experimental evidence has shown that charge-stabilized colloidal systems confined between glass plates show attractive interactions, while only repulsive interactions were expected to be present. Kepler and Fraden [1], were the first to report attractive interactions between charge-stabilized colloidal latex particles trapped between glass plates. They used $1.27 \mu \mathrm{m}$ particles and the video microscopy technique to observe the particles. From the images they obtained the pair correlation function. They obtained the interaction potential by an iterative method using Brownian dynamics simulations and a potential to extract the pair correlation function. The interaction stops when the calculated pair correlation function

4 Authors to whom any correspondence should be addressed. 
and the experimental one are matched. In this way, they reduced the influence of many-body interactions. They report the unexpected formation of an attractive well at distances of the order of the size of the particles. Later, Carbajal-Tinoco et al [2], using the same experimental geometry and technique, and $0.5 \mu \mathrm{m}$ particles, confirmed these results. These authors obtained the pair interaction potential by deconvoluting the experimental pair correlation function using a two-dimensional Ornstein-Zernike equation for several closures. Grier and co-workers [3] used a more idealized and simple system of confined colloidal particles between glass plates; they used optical tweezers to bring close together just two particles. In this way, many-body interaction problems can be avoided and the pair interaction potential can be extracted directly from the measured pair correlation function. They used $0.65,0.97$, and $1.53 \mu \mathrm{m}$ particles. These authors also observed the appearance of a secondary attractive well at large distances.

The existence of this unexpected secondary attractive well in the pair potential of confined colloids has received considerable attention - the aim is to find a satisfactory explanation of its nature [4-9] — but to date a full theoretical understanding of this phenomenon has not been achieved. A drawback in these confined colloidal systems between glass plates is the uncertainty that the plates introduce. Since glass plates become charged when they are in contact with water, it is expected that any theory attempting a full description of the experimental observations will have to take into account the electrostatic effects that the plates certainly exert on the particles.

A closely related and very interesting quasi-two-dimensional colloidal system is that formed at the air/water interface. In this system one avoids the uncertainties originating from the role of the glass walls. Pieranski [10] showed that charge-stabilized colloidal particles can be trapped at the air/water interface by surface tension effects, where part of the particle is submerged in the water but most of it remains exposed to the air. In this situation, only the part of the particle that is in contact with the water subphase gets a net surface charge, which is balanced by the counter-ions that swim around it. As a first approximation, Pieranski proposed that the charges at the particle and the counter-ions form a net dipole, which is perpendicular to the air/water interface. Therefore, the behaviour of colloidal particles floating on the water surface will be determined only by repulsive interactions. In this scenario, the interparticle distance will depend only on the surface density of particles. Amstrong et al [11], using charge-stabilized colloidal particles, performed careful experiments at high surface densities to study two-dimensional melting. They found evidence of defect-mediated melting and of the appearance of a hexatic phase. But they did not report the behaviour of colloids at the air/water interface at low surface densities.

More recently, experimental evidence has been presented which exhibits that not only repulsive interactions but also attractive interactions occur in quasi-two-dimensional colloidal systems at the air/water interface. Ruiz-Garcia and co-workers [12-15] have shown that colloidal particles trapped at the air/water interface form different patterns at lower surface densities, such as 2D colloidal clusters, voids, chains, and foams, as shown in figure 1 . The formation of these patterns cannot be explained solely on the basis of repulsive interactions. These experimental observations have been confirmed by other groups [16, 17].

Ruiz-Garcia and Ivlev [14] have presented a mechanism for explaining the formation of soap-froth and clustered structures in latex particles at the air/water interface. These authors proposed that the pair interaction potential must have an attractive component, that when competing with the repulsive electrostatic interactions can give rise to a secondary minimum; a deep primary minimum exists at short distances due to the conventional short-range van der Waals attractive interactions, when the distance between the surfaces of two particles is of the order of a few ångströms. Particles falling into the primary minimum lead to the formation of aggregates, dimers, trimers, etc, while particles falling into the secondary 

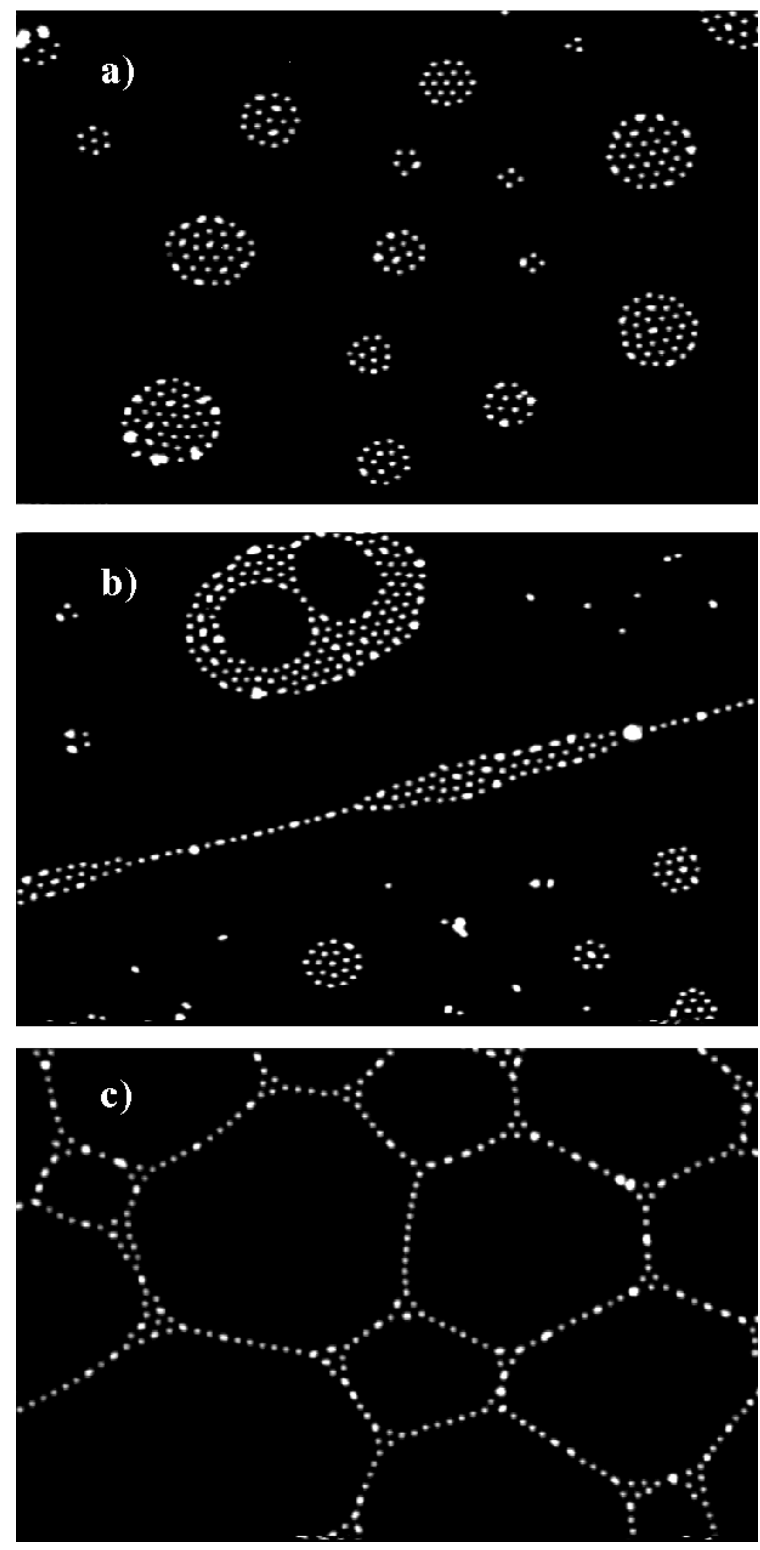

Figure 1. Optical microscopy images of $(a),(b)$ clusters, voids, and chains and $(c)$ foam-like structures formed by $1 \mu \mathrm{m}$ colloidal particles floating at the air/water interface.

minimum lead to clustering, where the equilibrium interparticle distance corresponds to the position of this minimum. Up to now, the interaction potential is analogous to that described by the Derjaguin-Landau-Verwey-Overbeek (DLVO) theory for three-dimensional colloidal systems [18], where competing short-ranged attractive and repulsive interactions occur. However, the DLVO theory cannot explain attractive interactions at the micron-size range, such as those observed in quasi-two-dimensional colloidal systems trapped between glass plates or at the air/water interface. 
In a $2 \mathrm{D}$ colloidal system at the air/water interface, while the origin of the electrostatic interaction can be regarded as of dipole-dipole type [10], the origin of the attractive interaction is not known. In addition, Ruiz-Garcia and Ivlev [14] assumed that the electrostatic interaction must be longer ranged (dipolar interaction), and this causes the interaction potential to oscillate (as a function of the interparticle distance), giving rise to a secondary maximum. This longrange repulsion plays an important role in the formation of colloidal chains, even though the interaction is completely isotropic.

In this paper, Monte Carlo (MC) computer simulation results are presented, for a model potential with the basic features of the interaction proposed by Ruiz-Garcia and Ivlev [14]. The potential used in the MC simulations was a discrete model given by a hard-sphere repulsion, combined with a first square shoulder (SS) representing the first repulsive barrier in real colloidal interactions, a square well (SW) to model the secondary minimum, and another SS to model the secondary maximum. This simulation study shows that this model potential is able to reproduce the patterns of colloidal particles at the air/water interface, validating the theory given in [14]. In order to compare the role played by the secondary maximum, we also present results for a potential with no secondary maximum at all, showing that a better qualitative agreement with the reported experimental observations is obtained when the potential has the second SS repulsion.

\section{Model potentials}

Systems of particles interacting via discrete potentials (DP) are useful as primitive models of liquids and colloids [19]. Arbitrary DP can be built via a sequence of SW and SS potentials, and, for example, Chapela et al [20] found via molecular dynamics simulations that very accurate DP of this type can mimic static and dynamic properties of true continuous Lennard-Jones fluids - but obtained in a more efficient way. Recently, other DP systems and their mixtures have been studied by means of perturbation theory and MC computer simulations [21,22].

In order to investigate the effect on the formation of meso-structures in two-dimensional colloidal systems due to a secondary minimum and a secondary maximum in the pair interaction potential, we performed MC computer simulations in the $N V T$ ensemble for several models of DP. We report here results for the DP models depicted in figures 2( $a$ ) and $(b)$, which will be referred to from now on as the DP-I and DP-II models. Both systems have a square shoulder of range $2 \sigma$ and height $5 \mathrm{kT}$, and a SW attractive interaction with a fixed width of $0.5 \sigma$ and depth $-1.5 k T$. In the case of the DP-II model, a second repulsive barrier of height $0.1 k T$ and variable range $\lambda_{r}$ is present when the distance between particles is greater than $3 \sigma$. The latter potential is able to reproduce several patterns formed by colloidal particles at the air/water interface as we will discuss now.

\section{Results and discussion}

The $N V T$ runs were made for systems with 208 and 512 particles, for three different densities covering the low-, medium-, and high-liquid-density region $\left(\rho^{*}=0.08,0.3\right.$, and 0.5$)$, and for three different temperatures $\left(T^{*}=k T=0.5,1\right.$, and 1.5), where $\rho^{*}=\rho \sigma^{2}$ is the reduced density and $\sigma$ is the diameter of the particles. Equilibrium configurations required between $1 \times 10^{5}$ and $5 \times 10^{7}$ cycles, depending on the values of the density and the temperature, in order to stabilize the configurational energy.

Final configurations for the DP-I model are shown in figure 3. The formation of chains at $\rho^{*}=0.5$ requires very long runs, more than $5 \times 10^{7}$ cycles, in order to give 

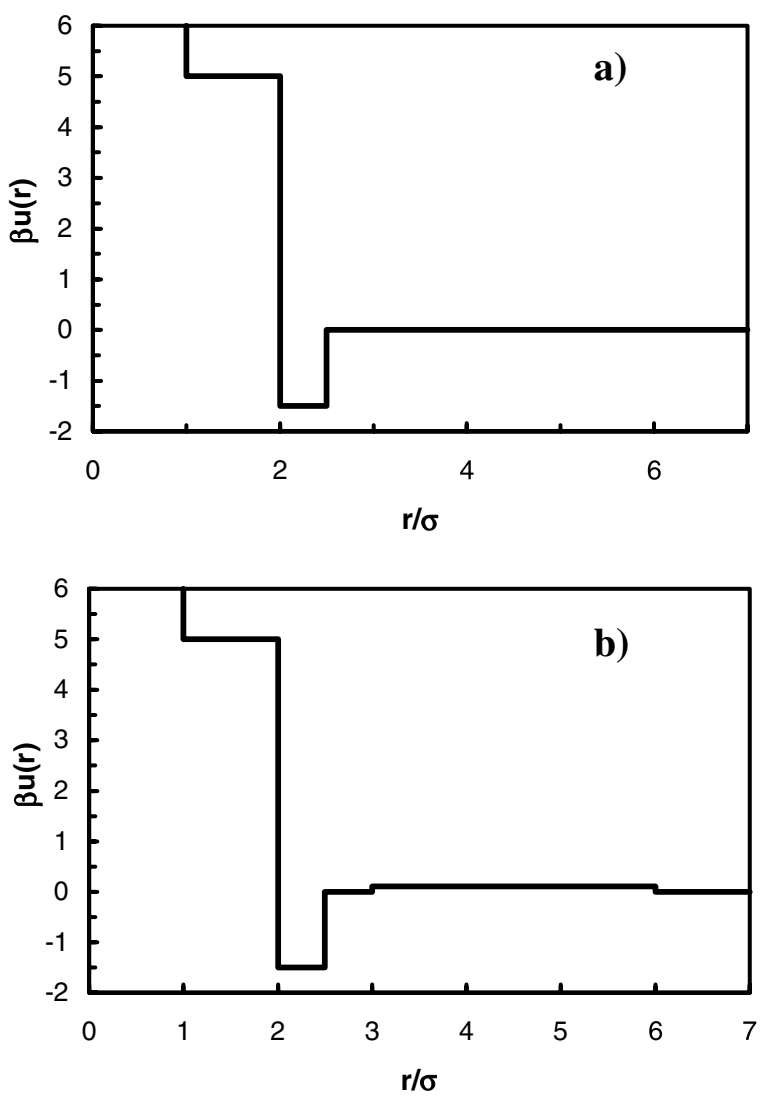

Figure 2. Pair interaction DP models used to simulate the behaviour of colloidal particles at the air/water interface. (a) The DP-I model contains a secondary minimum and $(b)$ the DP-II model contains a secondary minimum and a secondary maximum. Both models contain a square shoulder at shorter distances at $5 k T$.

stabilized configurational energies. However, in the case of longer-ranged potentials the energy stabilization was easily reached and similar structures were found. For $\rho^{*}=0.08$ and $T^{*}=0.5$, particles are arranged in a triangular lattice, where the lattice parameter corresponds to the position of the potential's minimum. The apparent structures in this case, represented in figure 3(a), are not really clusters but rather a single crystal, since the system is defined with periodic boundary conditions, and the non-connected structures in the box shown are indeed connected in the image boxes. Crystallization is absent at higher temperatures $\left(T^{*}=1\right.$ and 1.5), since the thermal energy is sufficiently high to destroy the ordering and increase the average interparticle distance, but remnants of the triangular lattice are still present in small regions. On the other hand, the minimum distance between particles is almost bigger than $2 \sigma$, as would be expected for a system of dilute hard discs whose diameter is twice that of the particles used here.

At $\rho^{*}=0.3$, and $T^{*}=0.5$. a very defective triangular lattice fills the entire simulation box; see figure $3(d)$. The competition between the repulsive and the attractive parts of the potential is such that the formation of short chains is frequent, where the distance between first neighbours is less than $2 \sigma$; the length of the chains becomes even bigger as the temperature is 

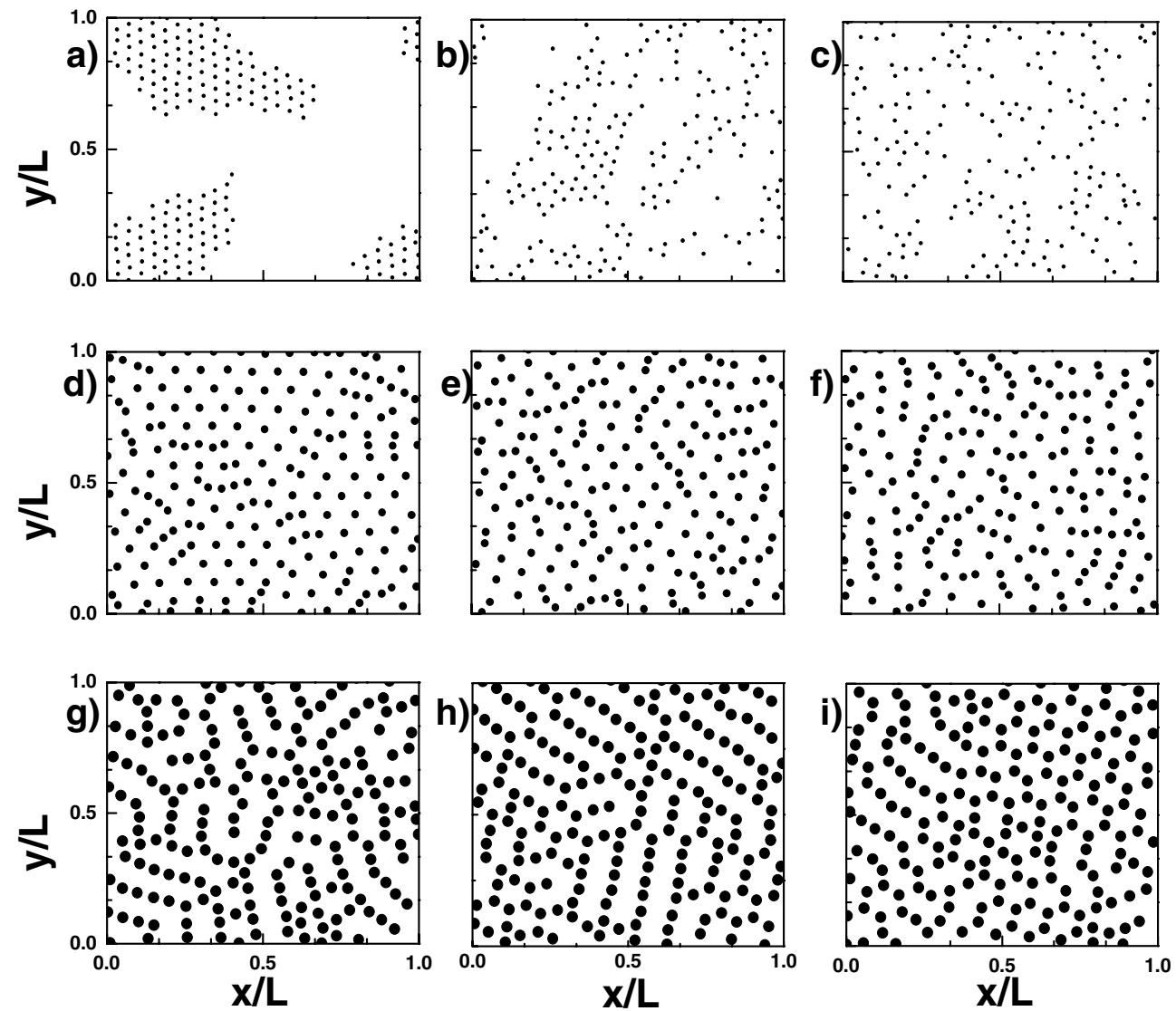

Figure 3. Final configurations obtained from the DP-I model for a system of 208 particles at densities $\rho^{*}=0.08,0.3$, and 0.5 (top to bottom), and temperatures $T^{*}=0.5,1.0$, and 1.5 (left to right), respectively; see the text for a discussion.

increased, as can be seen in figures $3(e)$ and $(f)$. At $T^{*}>0.5$, the system still retains a weak sixfold orientational order, interrupted frequently for the formation of large chains.

In figure 4, snapshots of the configurations for the DP-II model are presented for $\lambda_{r}=6 \sigma$, for the same thermodynamic states as were studied with the DP-I model, i.e., without a second long-ranged repulsive barrier. It can be noted that, for the low-density $\left(\rho^{*}=0.08\right)$ and lowtemperature $\left(T^{*}=0.5\right)$ state, particles arrange themselves in clusters, most of them of the same size ( $\sim 35$ particles on average). The main difference observed with respect to the same simulated state for the DP-I system is this clustering effect, due to the long-ranged repulsive barrier. This result indicates that the presence of this repulsive barrier is the determinant feature in the formation of clusters, as Sear et al [23] also obtained using a continuous potential with a long-range repulsion at large distances. At higher temperatures the clustering effect is dramatically reduced and kept at the level of chain formation, and well-defined empty regions, as voids, are present. These configurations of voids bordered by chains of particles resemble the soap-froth structures observed experimentally. At higher density $\left(\rho^{*}=0.3\right)$, chains are observed, but with an interparticle distance smaller than $2 \sigma$ inside the chains; see figures $4(d)-(f)$. This means that at higher densities the effect of the long-range repulsive barrier is diminished. Also notice that at higher temperatures the chain-like structures remain 

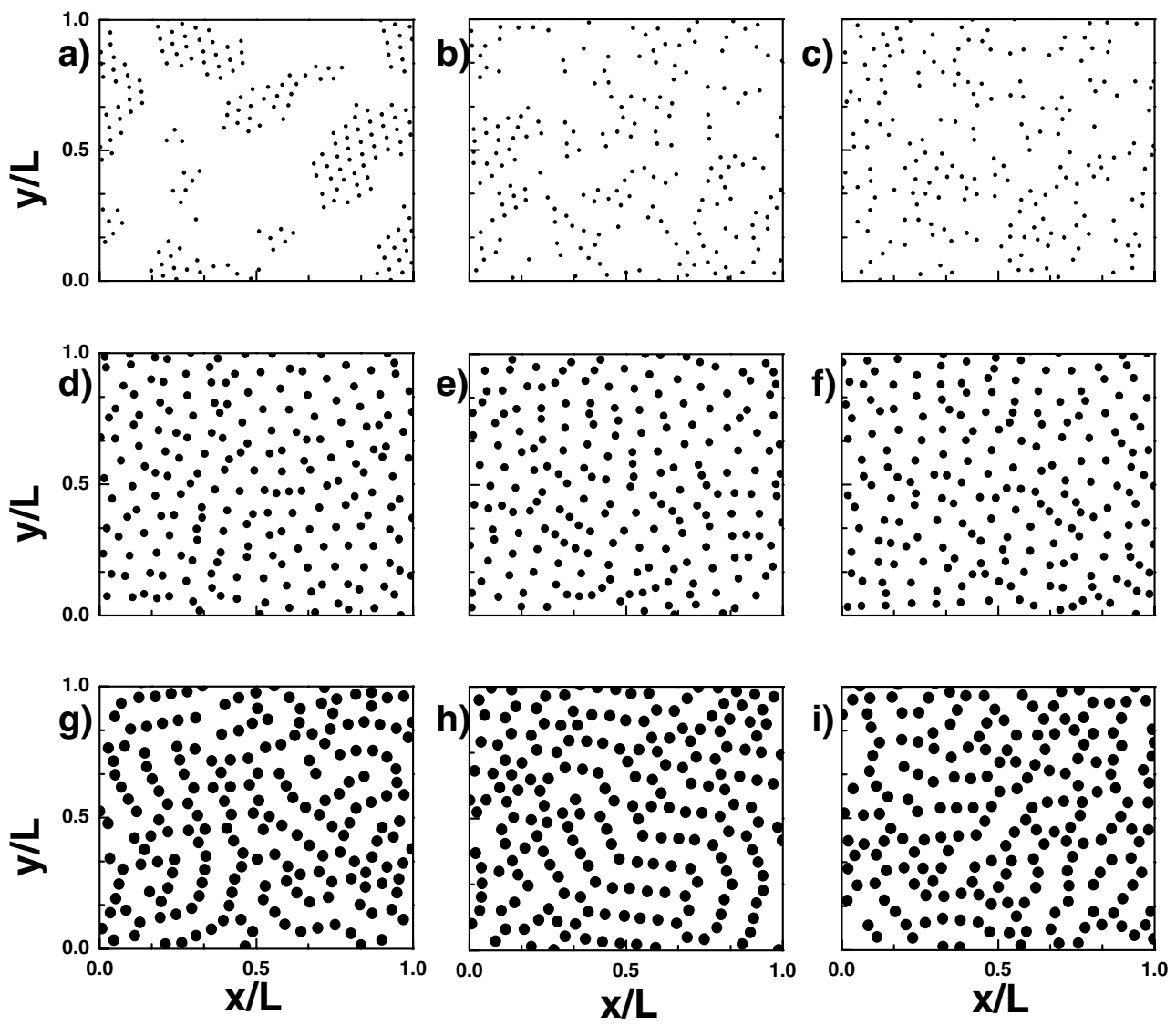

Figure 4. Final configurations obtained from the DP-II model for a system of 208 particles at densities $\rho^{*}=0.08,0.3$, and 0.5 (top to bottom), and temperatures $T^{*}=0.5,1.0$, and 1.5 (left to right), respectively. See the text for a discussion.

stable. On approaching a density as high as $\rho^{*}=0.5$, the sixfold structures are not present any longer, and instead we observe large chain-like structures forming labyrinthine patterns. These structures are consequence of the interplay between the primary maximum at $\sigma<r<2 \sigma$ and the SW attraction at $2 \sigma<r<3 \sigma$. Similar structures have been observed in systems of latex particles lying at the air-water interface, at similar densities.

If we consider a larger value of $\lambda_{r}$, for example $\lambda_{r}=7 \sigma$, then the most remarkable differences observed with respect to the DP-II system with $\lambda_{r}=6 \sigma$ were found at low densities $\left(\rho^{*}=0.08\right)$, where the size of the clusters is smaller, of the order of 20 particles on average per cluster (not shown), and they tend to arrange themselves in a triangular lattice at low temperatures. At higher densities, the patterns observed are similar to those shown in figures $4(d)-(i)$, with the difference that in the case of the longer-ranged potential the voids are better defined.

Studying the DP-II, $\lambda_{r}=7 \sigma$, system for 504 particles we found that there is no size dependence effect of the patterns observed for smaller systems. Since dense systems become hard to equilibrate, these simulations were carried on for $1.5 \times 10^{7}$ cycles. The pair correlation functions for a density $\rho^{*}=0.5$ and temperatures $T^{*}=0.5,1.0$, and 1.5 are presented in figure 5. As expected, the particles show a correlation at short distances, $1 \sigma \leqslant r \leqslant 2.5 \sigma$, which 


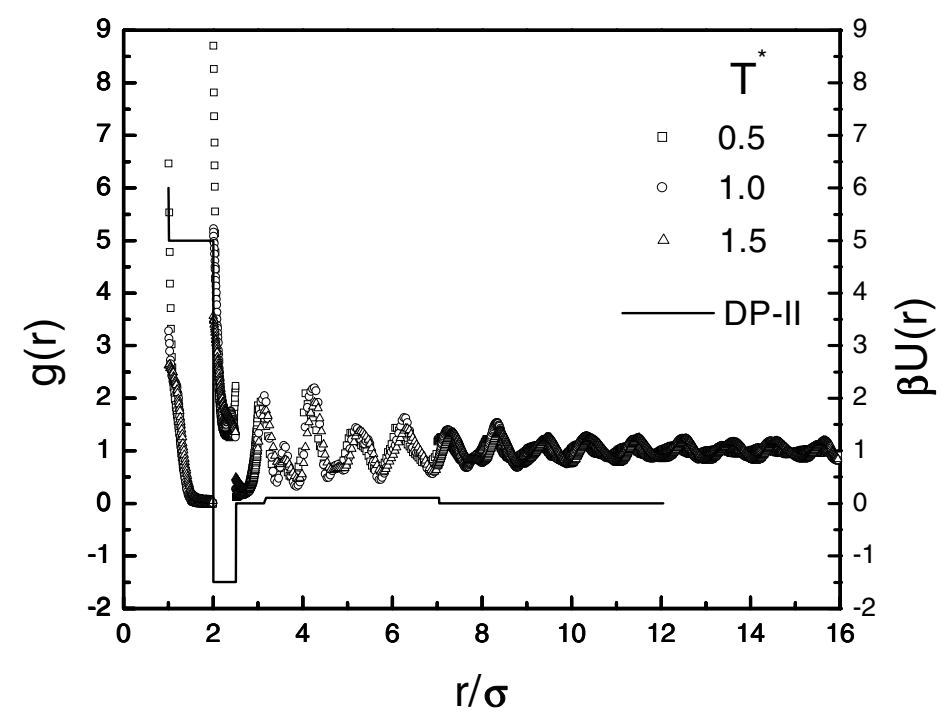

Figure 5. Pair correlation functions for a system of 504 particles interacting via the DP-II model with $\lambda_{r}=7 \sigma$ obtained at $\rho^{*}=0.5$ and $T^{*}=0.5,1.0$, and 1.5 .

is stronger at lower temperature; many of the particles remained at distances corresponding to the $5 k T^{*}$ shoulder, but most of them were located at distances corresponding to the secondary minimum. The $g(r)$ curves show that the system at the three temperatures studied has a well-defined structure, with a correlation much longer than that of a liquid phase. Another interesting characteristic of these curves is related to the two maxima present at interparticle distances of $r=3.1 \sigma$ and $4.2 \sigma$. Inspection of the corresponding configurations allows us to conclude that the peak at $r=3.1 \sigma$ is related to the number and length of the chains formed, since chain formation increases the frequency of third neighbours. On the other hand, the high peak at $r=4 \sigma$ is related to the high number of parallel chains, each pair of chains being separated by a distance slightly bigger than $2 \sigma$, such that, if the number of parallel chains is greater than 2, the number of next-neighbour chains separated by a distance $r=4 \sigma$ becomes significant.

Preliminary results on the phase diagrams of the two DP-II systems presented above have also been obtained by $N P T$-ensemble MC simulations, using the $N V T$ analysis reported in this paper [24]. Very rich phase diagrams are observed, with vapour-liquid and several liquid-liquid coexistence regions. The textures observed are also similar to the ones presented here. For example, in figure 6(a) a snapshot of an NPT run is shown, using 208 particles. The corresponding thermodynamic state is $T^{*}=0.5$ and $\rho^{*}=0.18$. In this state, the system shows the formation of voids inside a triangular lattice where the interparticle separation lies in the distance range that corresponds to the SW in the potential. The lattice and voids together form a sponge-like structure that resembles the early stages of the formation of soapfroths in a system of colloidal particles. Nevertheless, the dense regions in the simulation box appear to be highly ordered, as can also be seen in figure 6 where the radial distribution function is presented. The highest peak in $g(r)$ at $r=2 \sigma$ lies inside the potential well, as expected, and the next two peaks correspond to the distance between second neighbours and between third neighbours in a regular triangular lattice ( $r=3.9 \sigma$ and $4.5 \sigma$, respectively). The radial distribution function obtained for this density closely agrees with the experimental 


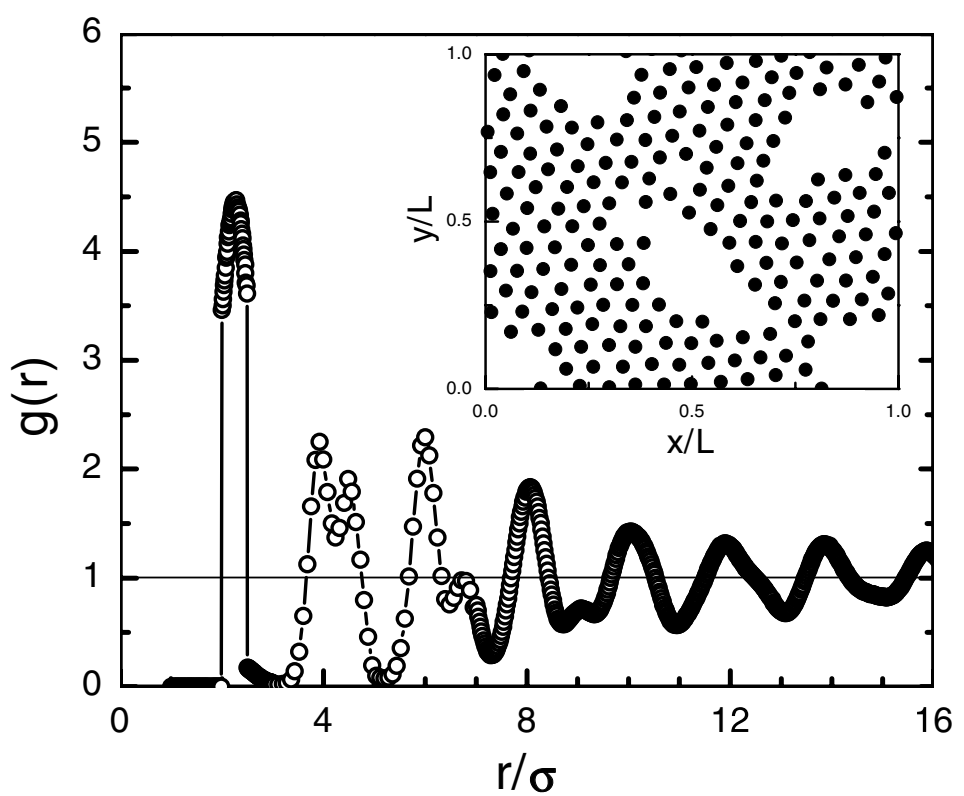

Figure 6. An NPT run for the DP-II model, a snapshot (inset) at $T^{*}=0.5$ and $\rho^{*}=0.18$, and its corresponding pair correlation function. Note the void or sponge-like structure formation.

$g(r)$ recently reported by Hansen et al [25]. These authors have shown the use of Delaunay triangulation to determine the distribution of pores and defects in colloidal films. This method can also be implemented in computer simulation studies, in order to provide a quantitative estimation of the distribution of voids observed in the configurations. The characterization of the phase diagram and the different fluid-like structures observed, including the distribution of voids, using the Gibbs ensemble computer simulation technique, will be reported in a future communication.

\section{Conclusions}

Stabilization of colloidal particles both in soap-froths and clusters at interparticle distances of the order of microns can not be interpreted solely in terms of the existence of a secondary minimum in the pair interaction potential, separated from the contact minimum (i.e., where van der Waals interactions dominate) by a repulsive maximum.

In this paper, we have shown the effect of the interplay between an attractive interaction at micron-size interparticle distances $r$ and a longer-ranged repulsive barrier at greater $r$. We have found by $N V T$ and preliminary NPT simulation studies that the clustering and soap-frothlike structures obtained resemble experimental observations, in agreement with the theory proposed by Ruiz-Garcia and Ivlev [14].

\section{Acknowledgments}

This work was supported by CONACYT (grants 28439E and ER026), IMP Molecular Engineering Programme and FAI-UASLP. 


\section{References}

[1] Kepler G M and Fraden S 1994 Phys. Rev. Lett. 73356

[2] Carbajal-Tinoco M D, Castro-Román F and Arauz-Lara J L 1996 Phys. Rev. E 533745

[3] Croker J C and Grier D G 1996 Phys. Rev. Lett. 771897

Larsen A E and Grier D G 1997 Nature 385230

Grier D G 1997 Curr. Opin. Colloid Interface Sci. 2264

Grier D G 1998 Nature 393621

[4] Sogami I and Ise N 1984 J. Chem. Phys. 816320

[5] Bowen W R and Sharif A O 1998 Nature 393663

[6] Neu J 1999 Phys. Rev. Lett. 821072

[7] Sader J E and Chan D Y 1999 J. Colloid Interface Sci. 213268

[8] Squires T M and Brenner M P 2000 Phys. Rev. Lett. 854976

[9] Ivlev B I 2001 Preprint cond-mat/0109487

[10] Pieranski P 1980 Phys. Rev. Lett. 45569

[11] Amstrong A J, Mockler R C and O’Sullivan W J 1989 J. Phys.: Condens. Matter 11707

[12] Ruiz-Garcia J, Gamez-Corrales R and Ivlev B I 1997 Physica A 23697

[13] Ruiz-Garcia J, Gamez-Corrales R and Ivlev B I 1998 Phys. Rev. E 58660

[14] Ruiz-Garcia J and Ivlev B I 1998 Mol. Phys. 95371

[15] Mejía-Rosales S J, Gamez-Corrales R, Ivlev B I and Ruiz-Garcia J 2000 Physica A 27630

[16] Ghezzi F, Earnshaw J C, Finnis M and McCluney M 2001 J. Colloid Interface Sci. 238433

Ghezzi F and Earnshaw J C 1998 Nuovo Cimento D 202243

Ghezzi F and Earnshaw J C 1997 J. Phys.: Condens. Matter 9 L517

[17] Pieranski P 1995 private communication

[18] Israelachvili J 1992 Intermolecular and Surface Forces (London, UK: Academic Press)

[19] Poon W C K and Pusey P N 1995 Observation, Prediction and Simulation of Phase Transitions in Complex Fluids (NATO ASI Series C, vol 460) (Dordrecht: Kluwer)

[20] Chapela G A, Scriven L E and Davis H T 1989 J. Chem. Phys. 914307

[21] Benavides A L and Gil-Villegas A 1999 Mol. Phys. 971225

[22] Vidales A, Benavides A L and Gil-Villegas A 2001 Mol. Phys. 99703

[23] Sear R P, Chung S, Markovich G, Gelbart W M and Heath J R 1999 Phys. Rev. E 59 R6255

[24] Mejía-Rosales S J 2000 Two-dimensional systems: colloidal particles at the air/water interface and vortices in superconducting films PhD Thesis Instituto de Física 'Manuel Sandoval Vallarta', Universidad Autónoma de San Luis Potosí, Mexico

[25] Hansen P H F, Rodner S and Bergstrom L 2001 Langmuir 174867 